\title{
Repercussions of Coronavirus on Cyber-Security Threats
}

\author{
Aryan Pandharipande ${ }^{1^{*}}$ and Ritik Parashar ${ }^{2^{*}}$ \\ ${ }^{1,2}$ Department of Computer Science and Engineering, Shri Ramdeobaba \\ College of Engineering and Management, Nagpur, India
}

\section{ABSTRACT}

Coronavirus outbreak has posed different challenges to society all over the world. The term 'new normal' has emerged and resulted in a change in the working style of society. Countries are using all of their resources to fight the virus. To keep the economy stable, to maintain the continuity, organizations are working from home with limited cyber-security. There is a sense of fear and hysteria among the people about the virus. Such situations are always taken into an advantage by cyber-criminals and the number of cyber- security threats has increased in the pandemic. This paper provides knowledge about cyber-security attacks on people during the COVID'19 pandemic. It provides details about various types of cyberattacks experienced by people, which people were affected by the threats, reasons behind their vulnerability. We throw light upon specifically the range of cyber attacks and their impact on the workforce. It provides details about how the attacks should be prevented in the future. This paper helps people understand the severity of the attacks, and how these are exercised. We aim to encourage people to remain vigilant against such breaches and prepare them to confront if they witness these types of attacks. The paper is also written with an intention to motivate and support further research related to cyber-crimes during the Coronavirus pandemic.

\section{KEY WORDS: COVID'19, CORONAVIRUS, WORKING FROM HOME, CYBER-CRIMES, CYBER-SECURITY THREATS.}

\section{INTRODUCTION}

The world has changed. Society has undergone a massive revolution in the year 2020. Novel Coronavirus, also called COVID'19 was first found in Wuhan province of China, at the end of the year 2019. Eventually, it spread across the world. On 11th March 2020, the World Health Organization (WHO) declared the virus as a global pandemic. The virus had a dramatic impact on society, which forced countries to impose 'lock-downs'. Various organizations and companies faced unusual challenges due to the pandemic. People found it difficult to organize

\section{ARTICLE INFORMATION}

*Corresponding Author: pandharipandeap@rknec.edu Received 30th Oct 2020 Accepted after revision 28th Dec 2020 Print ISSN: 0974-6455 Online ISSN: 2321-4007 CODEN: BBRCBA

Thomson Reuters ISI Web of Science Clarivate Analytics USA and Crossref Indexed Journal

\section{Clarivate
Analytics}

NAAS Journal Score 2020 (4.31)

A Society of Science and Nature Publication,

Bhopal India 2020. All rights reserved.

Online Contents Available at: http//www.bbrc.in/

Doi: http://dx.doi.org/10.21786/bbrc/13.14/11 their personal as well as professional lives. Term 'new normal' was coined due to the change in lifestyle of people, where organizations were working from home, to remain operable. This also brought a change in the working style of employees, who worked within their comfort zone. People got more involved with technology, but this change came with new cyber risks. It has been observed that the probability and consequences of cyber threat rose in the pandemic, because people did not take cyber-security practices seriously, and organizations were becoming more dependent on digital technology. It has been observed that there is an increase of around 6,000\% in spams that were related to the Coronavirus, according to IBM X-Force. Predominantly, people working from home, with limited cyber-security have fallen prey to these scams. Cyber-crime is a criminal offense in which either a computer or a computer network device is targeted or is used as a source. Cyber-criminals may perform both types of cybercrimes simultaneously. They target systems through viruses at the beginning and use those viruses to spread malware to different systems or through different network medium. Malware is short for Malicious Software. Some of the major malware are:

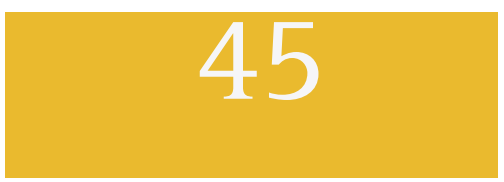


1. Virus: It is usually present in an executable file.

2. Trojans: It conceals itself within authorized software.

3. Worms: It infects the whole network of devices by replicating itself.

During the pandemic, the cyber-criminals also attacked health institutions, providing misinformation and fake news on COVID'19 cases. Hackers created fake URLs, providing nothing but fake news and malicious software that would infect the system. Cyber-criminals also distributed fake COVID'19 alerts as well as spread false emails disguising as video calling sites such as Zoom, Google Meet, etc. A lot of Corona virus-related domains are getting created daily. The malicious links infected the systems and created havoc. The Cybercriminals are taking advantage of the fear of the virus and uncertainty amongst people and using it as bait. There has been a new trend of 'Fearware', where these hackers are attacking through malware and ransomware with the undue advantage of Coronavirus. Bank related frauds have also increased like credit card skimming, including the introduction of Bank malwares such as Anubis Bankbot in malware related to Corona. A new trend of Zoombombing, i.e unwanted intrusion of people in a video conference with an intension of disruption has also taken a toll.

The structure of our paper is as follows: Section II contains Materials used. It describes in detail the types of cyber-attacks, such as Phishing, malware attack, etc. experienced by people. It explains the reason behind the increase in cyber attacks during the COVID'19 pandemic. It briefly describes the target audience. Section III contains Result and Discussion. It provides insights on the impact of the cyber-security threats to people in COVID'19 and what measures can be taken to prevent such threats. Section IV concludes the paper and summarizes the topic.

\section{MATERIAL AND METHODS}

Literature survey: Cyber attacks are mostly criminally or politically driven even though, some of the hackers relish to hack computer systems for their entertainment or for a sense of achievement. Politically persuaded cyber-attacks may take place to spread propaganda. With this, they intend to tamper the image of a specific government in the intellect of the citizens. Cyber-attacks may try to achieve destructive outcomes like to disclose sensitive information, confidential communication, or tampering the data. Due to the Coronavirus outbreak, several countries imposed lockdown, which forced organizations to work from home. This made the people turn towards technology for office work, communication and in short, stay connected.

This also resulted in the discontinuance of regular IT support and security to employees which they received in their offices. Many people who were not accustomed to the advances in technology had no other choice but to learn and use it. Cyber-criminals took the advantage of fear amongst the users about Corona and used it as bait. Various Cyber-security threats are taking place in the pandemic era, which includes email spamming, creation of malicious domains, the spread of malware, ransomware attack, BEC (Business Email Compromise), and many more. Threat Actors have always used the opportunity such as this pandemic to implement social engineering (taking advantage of errors by the human to extort sensitive information) techniques. We will see some of the cyber-security threats taking place in the COVID'19 pandemic in detail.

Phishing: Phishing is a cyber attack, which impersonates itself as an email. The email message tricks the user to believe it as an authentic and important email such as banks. There is an attachment with the mail, which if downloaded, malware gets triggered into the system. Phishing also has a goal of obtaining sensitive data of users like passwords. There have been various phishing scams since the outbreak of coronavirus. People are stressed due to the ongoing pandemic and are in constant fear. Any news related to the virus attracts people. The cyber-criminals have taken advantage of the anxiety and fear of people towards the virus. Most of the hackers have targeted health organizations, providing fake news about the pandemic.

Due to lockdown, people have turned towards web conferencing platforms like Zoom, Skype, etc. to continue their workflow. Taking advantage of this, fake notifications of zoom meetings, false COVID-19 alerts have become a common work of hackers. People, who get trapped into the web, end up clicking on the links, and the malware gets triggered into their systems. There have also been cases of fake Skype login pages, where login information collected sensitive information about the user. The reason behind the people falling prey to such emails and notification is their misunderstanding about how they are going to receive official news. According to a survey by IBM security and Morning Consult, around $46 \%$ of people think that COVID'19 related official news will be provided via email.

Malicious Websites: As the number of cases of Corona started increasing, people were panicking. The cybercriminals took advantage of the situation and started creating malicious websites, domains providing false news on COVID'19, etc. These are some of the websites which are found malicious and dangerous to visit.
1. Coronavirusstatus[dot]space
2. Coronavirus-map[dot]com
3. Bestcoronavirusprotect[dot]tk
4. Coronavirusupdate[dot]tk
5. Vaccine-coronavirus[dot]com

Banking Malwares: As the lockdown resulted in the shutdown of local shops and malls, more people started using online shopping on e-commerce websites. Hackers used the opportunity and used many non-authentic sites to gain information such as credit card details, passwords, etc. This led to credit card skimming. There has been a 
considerable rise in credit card-related fraud since the onset of lockdown. There are various banking malwares which have increased their features relating to Corona Virus. GINP is a banking Trojan which centrally targets Spanish banks. But during the pandemic, the authors introduced new features, called as 'Coronavirus Finder'. When one navigates over the URL, it gets redirected to a webpage called Coronavirus finder.The webpage shows the count of people infected with the Coronavirus around your location.

Figure 1: Graph depicting trends in daily domain registrations relating to COVID-19

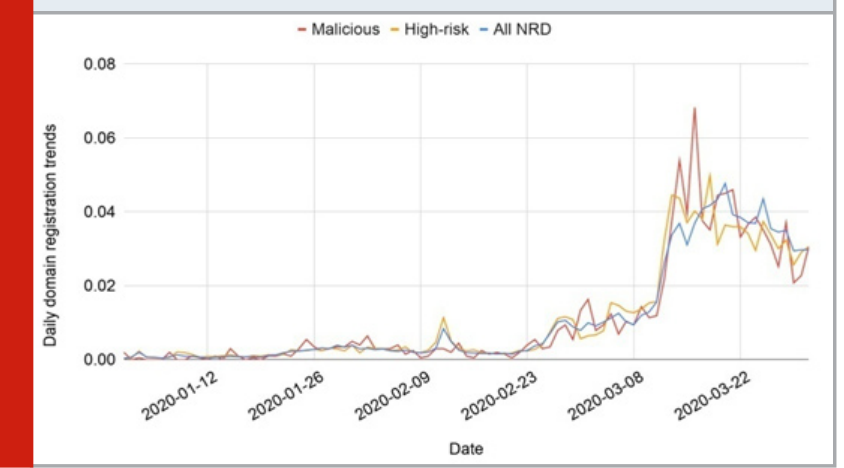

It asks users to pay some amount to know the exact location of the infected people. This tracks banking details of the users. An important thing to note that one needs to have GINP Trojan present in the system to open the webpage. Anubis Bankbot disguises as a COVID'19 related alerts application, but it only attracts users towards the application and collects banking details. Cerberus, also a Trojan, sends people an SMS which contains a link to download a malicious application known to track Corona Virus. People who believe the SMS end up downloading the application. With that, a Trojan also gets installed in the system which can steal personal information like username, password, banking details, etc.

Figure 2: Flowchart depicting trends in different types of Cyber-Security threats during COVID'19

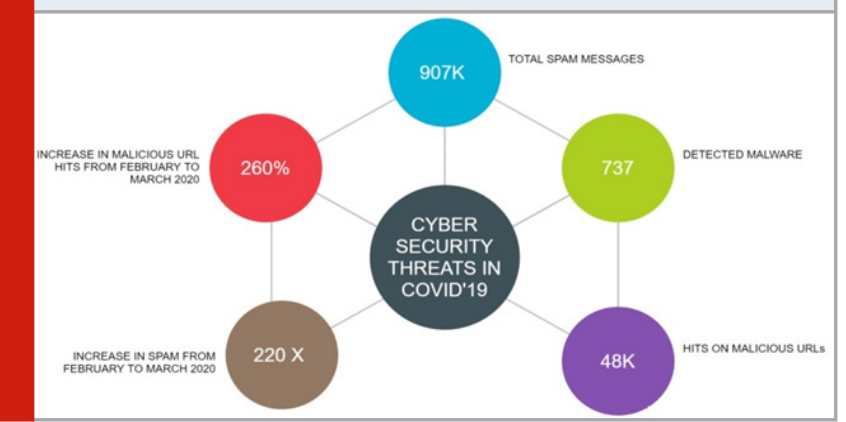

Other Malwares related to COVID'19: As discussed, people have been showing keen interest in any news or application relating to Coronavirus. People compromised cyber-security and downloaded various files that were nothing but malicious. Czech cyber-security agency (NUKIB) has published a detailed description of a
Coronavirus themed malware. The malware has a file called coronavirus installer. The malware makes the system unbootable. When it gets triggered, the machine restarts, and a Coronavirus themed page opens up with a message. Other functions like the exit button, opening task manager do not respond. When one restarts the system, a message pops up with a link to Discord, where the user can communicate with the hacker and find a solution in return for some ransom. Authors of a spyware known as 'AhMyth' have made an application on Google Play, which depicted coronavirus evolution. After installing the app, a few permissions are forced on the user for the functioning of the app.

As the permissions are given, malicious code runs and enables the creators to access the device, use the camera, click photographs, send and receive texts, know the location, etc. This is one of the most dangerous spying Trojan which has infected in the pandemic era. Vicious panda is a malware which targeted predominantly Mongolian sector. The creators usually send information related to COVID'19 through an RTF file. The main objective of the hackers is to spread RAT that is Remote Access Trojan in systems so that they can access information from the infected systems. Authors of this malware send a message related to a current hot topic, like Coronavirus. The criminals take advantage of preexisting vulnerabilities in the system and utilize them. After doing so, malware code gets triggered and provides remote access to the attackers. The attackers can have the ability to take Screenshots, Download files, delete files, get passwords and username, etc.

\section{RESULTS AND DISCUSSION}

Virtual Private Network (VPN) has become crucial as organizations are functioning online and students are learning in virtual classrooms, therefore, the major aim is the security and their ability to continue their work. Lack of preparedness in an organization will result in misalignment of VPN which may give away crucial information and even fall prey to Distributed Denial of Service (DDoS) attacks. Further to this, many users may use their personal computers to execute official work which could also lead to a great risk for companies and organizations. Misinformation has led to the wastage of a huge amount of time in the course of the pandemic. False information has a more adverse effect than having no formation at all. Almost every individual is required to include technology, therefore it is assumed that everyone is handy to technical skills. Big corporations' VPNs are not able to handle the burden to access the network and thus it has reduced their productivity while working from home. Organizations and individuals also need to take some preventive measures which can reduce their vulnerability.

1. One should avoid clicking on any UNKNOWN messages with links to install an application from mysterious sources.

2. Files should be backed up and stored in devices other than your system (e.g. on an external drive) and sensitive information should be protected. 
3. One should cautiously examine any URL or email address they see and download only from authentic sources.

4. One should ensure that they have legitimate and latest anti-virus software installed on their computer and mobile devices.

5. One should be aware of emails and messages that are asking for confidential information.

6. Administrations' vulnerabilities of the system that attackers could exploit should be secured.

7. Organizations are advised to reform at their BCPs and secluded working policies at the same time prioritizing cyber security within post COVID-19 re-strategizing operation.

8. One should always verify that they are on company's legit website before advancing their personal information.

\section{CONCLUSION}

Huge amount of change can be seen in criminal activity due to COVID-19. There has been a decrease in crimes such as burglary, pickpocketing but cyber crimes have increased as hackers have taken advantage of fear amongst people about COVID'19. Cyber-criminals are changing their strategy and taking advantage of the anxiety and hysteria amongst people about the virus. As we have seen, hackers made available applications about the pandemic, spread fake news which also came with malicious links, websites, or codes that infect the systems. Many people who work from home do not have adequate cyber-security measures to tackle such attacks. Also, it has been observed that not many people are taking cyber-security seriously. Such people are falling prey to the attack. At this particular time, the main focus should be on cyber-security education among citizens, as it is the most important thing needed right now. Organizations should look after their workforce as it is no more beneath the organization's firewalls, employees of organizations are working from home with limited security. Organizations should make sure to prepare their workforce, so they could protect themselves.

\section{REFERENCES}

Alexander Eremin," People infected with coronavirus are all around you, says Ginp Trojan.The Coronavirus Finder (that doesn't work)”, 24 Mar, 2020.

Beware of potentially dangerous banking trojan ‘Cerberus' exploiting COVID-19 crisis: CBI”, F E online,
19 May, 2020.

Coronavirus Disease (COVID-19) Pandemic”,World Health Organisation,Eroupe,Health Topics. Covid 19 Cyber attacks", Infosecawareness

C OVID-19 cyberthreats :Types of cyberattacks",Interpol

Cybercriminals love COVID-19: Here's how to stay cyber-secure",Telenor Mar, 2020.

Cybereason Nocturnus," Just Because Your at Home

Doesn't Mean Your Safe”,Cyberreason,18 Mar,2020

Cybersecurity Leadership Principles: Lessons learnt during the COVID-19 pandemic to prepare for the new normal, May”,World Economic Forum, 26 May 2020, pp-10.

David García, "Vicious Panda: A New Malware Campaign Exploiting Coronavirus”,Buguroo.

David Morán," Android Malware takes Advantage of COVID-19",Buguroo.

Developing Story: COVID-19 Used in Malicious Campaigns",Trend Micro, 11 Nov, 2020.

D0 Not Open These 14 Coronavirus Websites", Gagdets Now Bureau, 16 March, 2020. How Cyber Attacks Happen", Equifax

Janos Szurdi, Zhanhao Chen, Oleksii Starov, Adrian McCabe, Ruian Duan, "Studying How Cybercriminals Prey on the COVID-19 Pandemic", Paloalto Networks , Unit 42,22 April, 2020.

Jerome Segura, “Online credit card skimming increased by 26 percent in March”,Malware Bytes, 9 Apr, 2020.

Josh Fruhlinger," What is Phishing? How this cyber attack works and how to prevent it",CSO, 4 Sept, 2020.

Kelly Sheridan, "Why Consumers, SMBs Are Likely to Fall for Coronavirus Scams”,Dark Reading,23 Apr, 2020.

"Klaus Julisch, Florian Widmer, Michael Grampp, "Cyber crime - the risks of working from home", Deloitte.

Tope Aladenusi, “COVID’19's Impact On Cybersecurity”,Delloite, Mar, 2020,pp 1-3

Tips on how to protect yourself against cybercrime “,What is Cyber Crime, Kaspersky

Tom Kelly, “How Hackers are using COVID'19 to find new phishing victims,"Secuirtymagzine,23 Jun, 2020. "What is Malware", Forcepoint

What is Social Engineering", Kaspersky

Zoombombing", Wikepedia 